\title{
Measurement of Residual Chromatic Dispersion or OSNR via Nonlinear Spectral Evolution
}

\author{
Clément Courvoisier, Julien Fatome and Christophe Finot
}

\begin{abstract}
In this work, we report a new fiber architecture providing high accuracy measurements of both residual chromatic dispersion and optical signal to noise ratio. Taking advantage of the nonlinear spectral evolution of pulses propagating in a Kerr medium, we have numerically and experimentally validated the use an anomalous dispersive fiber combined with a central spectral filtering.
\end{abstract}

Index Terms - Optical performance monitoring, Nonlinear optics, Optical fiber, Optical Telecommunication.

\section{INTRODUCTION}

$\mathrm{W}$ ith the advent of high-speed optical networks and associated increasing repetition rates and complexity, it has become vital to benefit from accurate tools capable of measuring the various impairments that degrade the data stream. All-optical solutions enabling a direct performance monitoring is therefore a growing field of investigation in order to correct for the variation in link performance.

Among the large set of methods that have already been tested [1, 2], several techniques based on a nonlinear element have demonstrated their potential to monitor the level of residual chromatic dispersion (RCD) as well as the optical signal to noise ratio (OSNR). We can highlight several works taking advantage of semiconductors optical amplifiers [3] or nonlinear effects taking place in optical fibers, such as Brillouin backscattering [4] or Kerr nonlinearity. In the latter case, various configurations have been experimentally implemented : Kerr nonlinearity acting in a nonlinear optical loop mirror [5], through four-wave mixing process [6], through cross-phase modulation [7] or through self-phase modulation undergone in a normally dispersive fiber followed by an offset spectral filtering [8].

In this letter, we propose and experimentally demonstrate a new configuration enabling both monitoring of the RCD and OSNR of a return-to-zero single-wavelength signal. Compared to [8], our new scheme benefits from the selfphase modulation occurring in an anomalous dispersive optical fiber and requires a centered optical bandpass filtering (OBPF). In the first part of the article, we describe

Manuscript received October 3rd 2010

This work was supported by the Agence National de la Recherche (FUTUR - ANR-06-TCOM-016 - and PERSYST II - ANR-07-TCOM014 projects.) and by the Conseil Regional de Bourgogne. It benefits from the PICASSO platform.

Clément Courvoisier, Julien Fatome and Christophe Finot are with the Laboratoire Interdisciplinaire Carnot de Bourgogne, UMR 5209 CNRS Université de Bourgogne, 9 Av. A. Savary, BP 47870, 21078 Dijon Cedex, France (christophe.finot@u-bourgogne.fr). the principle as well as the experimental set-up of our device. In following sections, experimental and numerical results reveal a high sensitivity of our device relative to RCD or OSNR levels.

\section{EXPERIMENTAL SET-UP AND PRINCIPLE OF OPERATION}

Our experimental setup is sketched in Fig 1(a). The signal under test is made of a train of optical pulses emitted by a modelocked fiber-based laser (MLL) running at a repetition rate of $10 \mathrm{GHz}$. The ultrashort pulses have a temporal duration of 2.4 ps with a central wavelength of $1550 \mathrm{~nm}$. The RCD is emulated by means of an intensity and phase programmable optical filter (Finisar WaveShaper device) that enables to imprint a parabolic phase profile on the incoming pulses [9]. The resulting signal is then degraded by means of its combination with a spectrally filtered amplified spontaneous emission source (ASE). By tuning the level of spectral attenuation on the WaveShaper, a direct control of the OSNR is achieved.

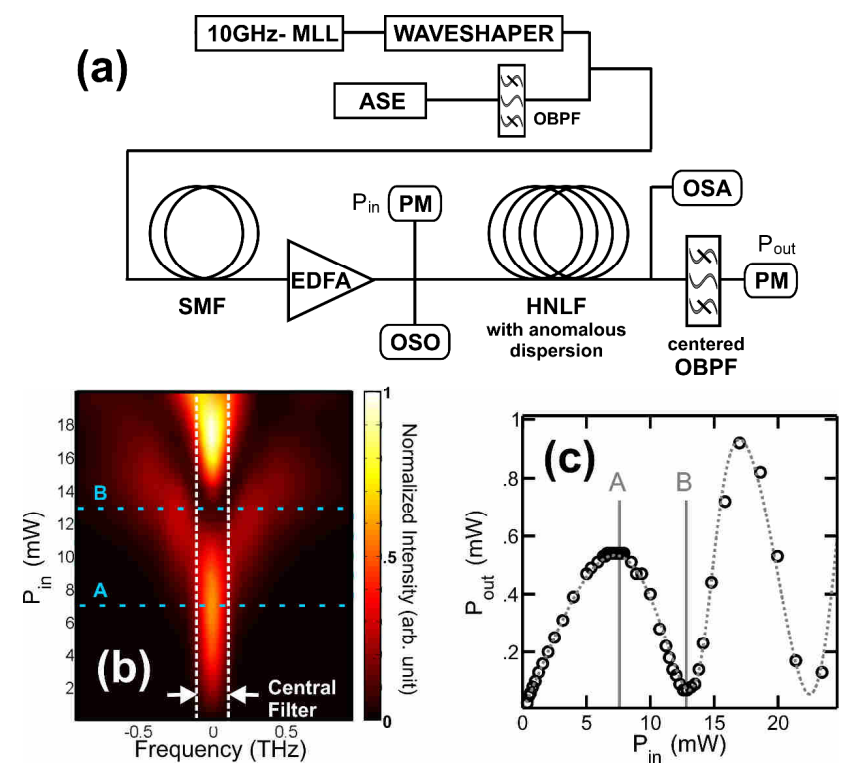

Fig. 1: (a) Experimental set-up. PM : powermeter (b) Evolution of the experimental optical spectrum recorded after the HNLF as a function of input average power. Dashed white line illustrates the FWHM of the OBPF. (c) Transfer function of the device: experimental results (circles) are compared with numerical simulations (dashed grey line).

The core of our optical monitoring device relies on a 920-m long highly nonlinear fiber (HNLF) with a Kerr coefficient of $10 \mathrm{~W}^{-1} \cdot \mathrm{km}^{-1}$ and an anomalous dispersion of $0.7 \mathrm{ps} / \mathrm{km} / \mathrm{nm}$. The linear losses and dispersion slope are both reduced and can be neglected. In order to reach an adequate working power (WP) and to observe the expected 
nonlinear behavior, the fiber is preceded by an erbiumdoped fiber amplifier (EDFA) and by an additional segment of standard single mode fiber SMF (31 m, the purpose of this segment will be discussed in section III).

Within the HNLF segment, pulses experience a significant spectral broadening linked to self-phase modulation as it is highlighted in the experimental spectral measurements depicted in Fig. 1(b). Note that this expansion is also characterized by a depletion of the central part of the spectrum for input average powers close to $13 \mathrm{~mW}$ (point B). For higher power levels, solitonic compression leads to general enrichment of the spectrum.

At the fiber output, an OBPF centered on the signal initial wavelength and characterized by a Gaussian shape with a full width at half-maximum (FWHM) of $100 \mathrm{GHz}$ carves into the resulting expanded spectrum.

A key parameter of our device is the shape of its transfer function (TF) that non-linearly maps the input average power to the average power obtained after this filtering. Indeed, according to the choice of fiber parameters, three behaviors can be expected : a monotonously increasing TF, a curve exhibiting a marked plateau or a raise and fall evolution [10]. Contrary to the applications usually targeting an all-optical ultrafast power-limiter [11, 12], we voluntarily implement here a strong rise and fall evolution that enables an enhanced sensitivity towards the initial pulse stream fluctuations. Such a feature has already been beneficial in the context of an ultrafast all-optical fiberbased amplitude jitter magnifier [13] as well as in a bit error indicating scheme [14]. The TF we experimentally measured is plotted in Fig. 1(c) (open circles) and is in remarkable agreement with the results obtained by means of simulations based on the numerical integration of the standard nonlinear Schrödinger equation (solid line). The central depletion of the spectrum observed for input powers close to $13 \mathrm{~mW}$ is responsible for the minimum of output power observed on the TF.

\section{ChrOMATIC DISPERSION MONITORING}

In this section, we now focus on the ability of our device to efficiently highlight the consequences of RCD (we consider in our discussion an input signal only affected by RCD and not by nonlinear impairments). The spectral phase imposed by the dispersion emulator directly impacts the temporal width of the pulses entering into the HNLF as can be seen in Fig. 2(a), where the temporal duration of pulses has been measured by an optical sampling oscilloscope, OSO (see for example insets of Fig. 2(a)). Let us note that the fixed 31-m long segment of SMF imposes an additional chirp on the incoming pulses, so that the minimum temporal duration was not obtained for a RCD equals to zero.

For a given average power, the amount of RCD directly influences the input peak power of the pulses so that the resulting spectral broadening is severely affected. Indeed, the self-phase modulation induced in the HNLF segment depends on the temporal gradient of the intensity profile, in other words, on both the peak-power and temporal duration. Fig. 2(b) illustrates such a dependence for an input average power of $7.25 \mathrm{~mW}$ (ie, working point corresponding to the point A on Fig. 1(c)).
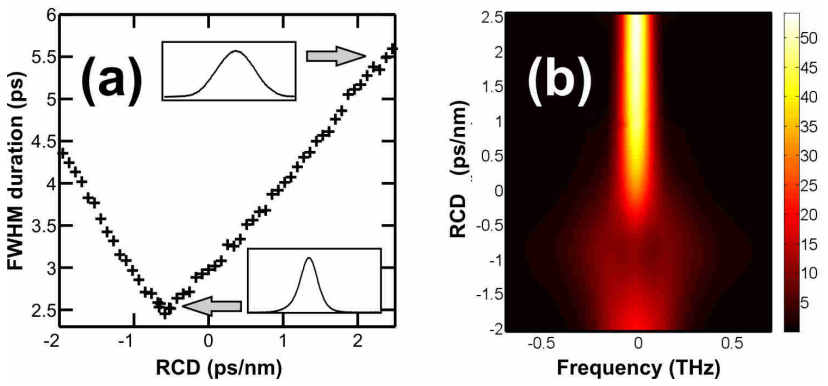

Fig. 2: (a) FWHM temporal duration of the pulse entering the HNLF according to the RCD induced by the dispersion emulator. Insets : temporal intensity profiles recorded over a time span of $15 \mathrm{ps}$. (b) Experimental demonstration of the influence of the RCD on the resulting spectrum recorded at the output of the HNLF and for a working average power of $7.25 \mathrm{~mW}$.

This sensitivity is also readily apparent on the $\mathrm{TF}$ : Fig. 3(a) summarizes the TF recorded for various amounts of RCD and highlights a continuous expansion of the TF towards higher average input powers. The experimental results, plotted in Fig. 3(a2) are once again in quantitative agreement with these numerical predictions and underline the high sensitivity of our device towards the RCD. Consequently, if for a practical use of our device we operate at a fixed input average power, we can make out that the output average power will be strongly affected by the level of RCD (Fig. 3(b)). To illustrate this point, we have considered the evolution of the output average power as a function of the RCD for two different initial working powers, corresponding to the local extrema of the TF (with no $\mathrm{RCD}$ ): maximum $\mathrm{A}$ (black crosses) and minimum $\mathrm{B}$ (grey crosses) as defined in Fig. 1(c).
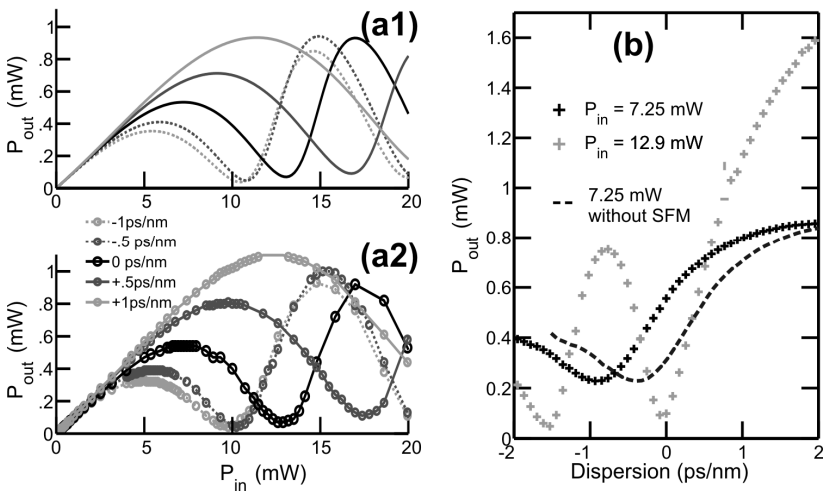

Fig. 3: (a) TF for various amounts of RCD. Numerical simulations (a1) are compared with experimental results (a2). (b) Influence of the RCD on the output power for two WPs : initial average power of $7.25 \mathrm{~mW}$ (black crosses) and $12.9 \mathrm{~mW}$ (grey crosses). The results without the SMF (numerical simulations) are plotted using a dashed black line.

In the case of the WP A $(7.25 \mathrm{~mW}$, black crosses $)$, we can make out a range from -1 to $2 \mathrm{ps} / \mathrm{nm}$ for which the evolution of the output power according to the level of RCD is monotonously increasing. Moreover, in this span, one can not only get access to the absolute value of the RCD, but also to its sign, which was not obvious with previously demonstrated methods and which is crucial to ensure a convenient feedback to a tunable dispersion compensator. This extended range of unambiguous measure is closely linked to the insertion of the SMF pre-chirp: without such a fiber, the measurable RCD would have been restricted to -.3 
to $2 \mathrm{ps} / \mathrm{nm}$, as shown by results based on numerical simulations plotted with a dashed black curve in Fig. 3(b).

If we now operate at the WP B (12.9 mW, grey crosses), we can make out that our device would exhibit a higher sensitivity to the level of RCD. However, this is at the cost of the information regarding the sign of the RCD. In this context, combining the measurements at working powers $\mathrm{A}$ and $\mathrm{B}$ could be beneficial to achieve an accurate value of low RCD levels: WP A providing the sign of the RCD as well as a rough estimation, while the absolute value can be then finely determined using WP B.

\section{IMPACT OF OSNR}

In this final section, we investigate the impact of the in-band optical signal to noise ratio (OSNR) on the average output power. In this series of experiments, the OSNR is simply tuned by changing the level of signal mixed with the source of ASE, see Fig. 1(a). Results are summarized in Fig. 4(a) and stress that for a degraded OSNR, an additional input power is required to observe the same TF. This can be physically understood given the fact that, for a fixed temporal pulse profile, the SPM-driven spectral broadening is predominately sensitive to the pulse peak-power, directly related to the pulse energy. Consequently, a decreased OSNR will require a higher average power to compensate for the energy contained in the noise and therefore to maintain the same pulse energy. Therefore, point A does not exhibit significant change in the output power but requires a higher input average WP (this property could be useful for simultaneous monitoring of RCD and OSNR). More interestingly if the RCD is fixed, we can make out that by operating at WP B the output power is directly correlated to the OSNR. As shown by experimental results depicted in Fig. 4(b), the output power varies continuously from 0.1 to $0.55 \mathrm{~mW}$ for an OSNR ranging from -32 to $-15 \mathrm{~dB}$. Hence, a measurement of the output power can enable us to easily monitor the OSNR of the incoming signal.
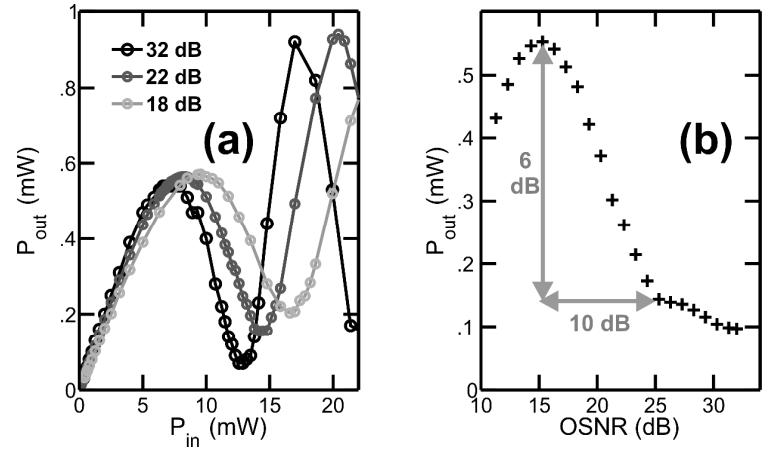

Fig. 4: (a) Influence of the OSNR on the TF. (b) Output average power as a function of the input signal OSNR for a working power of $12.9 \mathrm{~mW}$.

\section{CONCLUSION}

We have proposed and experimentally demonstrated a new practical all-optical architecture that provides a direct measurement of the OSNR or level of residual chromatic dispersion. The sensitivity of the device is remarkable and RCD with absolute value below $1 \mathrm{ps} / \mathrm{nm}$ can easily be detected. Our proof-of-principle experiments were based on a $10-\mathrm{GHz}$ 2.4-ps signal, but additional numerical simulations have revealed the possibility to extend this concept to higher repetition rates and other pulse durations (longer pulse durations can indeed enable a larger RCD measuring range). Based on a central filtering and anomalous dispersion regime, our setup has the advantage of requiring moderate input powers compared to other solutions. Moreover, the use of an anomalous segment of HNLF limits the detrimental pulse-to-pulse interactions [10]. Finally, the use of a fiber element could also be replaced in a near future by the implementation of highly nonlinear waveguides that would allow further photonic integration [15], the constant progress in dispersion management in this waveguide enabling to obtain anomalous dispersion.

\section{REFERENCES}

[1] Z. Pan, C. Yu, and A. E. Willner, "Optical performance monitoring for the next generation optical communication networks," Opt. Fiber. Technol., vol. 16, pp. 20-45, 2010.

[2] D. C. Kilper, R. Bach, D. J. Blumenthal, D. Einstien, T. Landolsi, L. Ostar, M. Preiss, and A. E. Willner, "Optical performance monitoring," J. Lightw. Technol., vol. 22, pp. 294-304, 2004.

[3] P. Vorreau, D. C. Kilper, and J. Leuthold, "Optical noise and dispersion monitoring with SOA-based optical 2R regenerator," IEEE Photon. Technol. Lett., vol. 17, pp. 244-246, 200.

[4] M. Pelusi, A. Fu, and B. J. Eggleton, "Multi-channel in-band OSNR monitoring using stimulated Brillouin scattering," Opt. Express, vol. 18, pp. 9435-9446, 2010.

[5] R. Adams, M. Rochette, T. T. Ng, and B. J. Eggleton, "All-optical inband OSNR monitoring at $40 \mathrm{~Gb} / \mathrm{s}$ using a nonlinear optical loop mirror," IEEE Photon. Technol. Lett., vol. 18, pp. 469-471, 2006.

[6] T. T. Ng, J. L. Blows, M. Rochette, J. A. Bolger, I. C. M. Littler, and B. J. Eggleton, "In-band OSNR and chromatic dispersion monitoring using a fibre optical parametric amplifier," Opt. Express, vol. 13, pp. 5542-5552, 2005.

[7] T. D. Vo, M. D. Pelusi, J. Schröder, B. Corcoran, and B. J. Eggleton, "Multi-impairment monitoring at $320 \mathrm{~Gb} / \mathrm{s}$ based on cross-phase modulation radio-frequency spectrum analyser," IEEE Photon. Technol. Lett., vol. 22, pp. 428-430, 2010.

[8] P. S. Westbrook, B. J. Eggleton, G. Raybon, S. Hunsche, and T.-H. Her, "Measurement of residual chromatic dispersion of a $40-\mathrm{Gb} / \mathrm{s} \mathrm{RZ}$ signal via spectral broadening," IEEE Photon. Technol. Lett., vol. 14, pp. 346-348, 2002.

[9] M. A. F. Roelens, S. Frisken, J. Bolger, D. Abakounov, G. Baxter, S. Poole, and B. J. Eggleton, "Dispersion trimming in a reconfigurable wavelength selective switch," J. Lightw. Technol., vol. 26, pp. 73-78, 2008.

[10] J. Fatome and C. Finot, "Scaling guidelines of a soliton-based power limiter for 2R-optical regeneration applications," J. Lightw. Technol., vol. 28, pp. 2552-2556, 2010.

[11] M. Asobe, A. Hirano, Y. Miyamoto, K. Sato, K. Hagimoto, and Y. Yamabayashi, "Noise reduction of $20 \mathrm{Gbit} / \mathrm{s}$ pulse train using spectrally filtered optical solitons," Electron. Lett., vol. 34, pp. 1135$1136,1998$.

[12] M. Gay, M. Costa e Silva, T. N. Nguyen, L. Bramerie, T. Chartier, M. Joindot, J. C. Simon, J. Fatome, C. Finot, and J. L. Oudar, "170 $\mathrm{Gbit} / \mathrm{s}$ bit error rate assessment of regeneration using a saturable absorber and a nonlinear fiber based power limiter," IEEE Photon. Technol. Lett., vol. 22, pp. 158-160, 2010.

[13] C. Finot and J. Fatome, "All-optical fiber-based ultrafast amplitude jitter magnifier," Opt. Express, vol. 18, pp. 18697-18702, 2010.

[14] S. Oda and A. Maruta, "Two-bit all-optical analog-to-digital conversion by filtering broadened and split spectrum induced by soliton effect or self-phase modulation in fiber.," IEEE J. Sel. Top. Quantum Electron., vol. 12, pp. 307-314, 2006.

[15] M. Pelusi, F. Luan, T. D. Vo, M. R. E. Lamont, S. J. Madden, D. A. P. Bulla, D.-Y. Choi, B. Luther-Davies, and B. J. Eggleton, "Photonic-chip-based radio-frequency spectrum analyser with terahertz bandwidth," Nature Photonics, vol. 3, pp. 139-143, 2009. 